Conclusions Almost 20\% of first-year pre-professional dancers reported an ankle injury, with more than $80 \%$ of these injuries leading to dance time-loss. No significant risk factors could be identified for ankle injuries throughout the academic year. Since ankle injures are common injuries among dancers, research in larger dance populations with longer term of follow-up, including relevant risk factors, is necessary to evaluate the impact of these injuries further.

\section{EPIDEMIOLOGY OF ANKLE SPRAINS IN ELITE HIGH SCHOOL BASKETBALL PLAYERS: MEDICAL AND PHYSICAL CHECKUP REPORT, RELATIONSHIPS BETWEEN PREVALENCE OF ANKLE SPRAINS, UNSTABLE SENSATION, ATFL PATHOLOGY AND THE BALANCE TEST}

\footnotetext{
${ }^{1,2}$ Shuichi Nakayama, ${ }^{2}$ Tsukimura Naoki, ${ }^{2}$ Iwakura Nahoko, ${ }^{2}$ Yamamoto Takayuki, ${ }^{2}$ Saku Isaku, ${ }^{2}$ Ito Eri, ${ }^{2}$ Takebayashi Tomomi, ${ }^{2}$ Uchino Sayuri, ${ }^{3}$ Numasawa Shun, ${ }^{3}$ Arimoto Kumi, ${ }^{3}$ Ikeda Shigehiro. 'JR Tokyo General Hospital, Tokyo, Japan; ${ }^{2} J a p a n$ Basketball Association, Tokyo, Japan; ${ }^{3}$ Osaka Basketball Association, Osaka, Japan
}

\subsection{6/bjsports-2021-IOC. 104}

Background Ankle sprains are the most common injury among basketball players. However, a number of athlete report persisting symptoms which can lead to the chronic ankle instability (CAI), even worse, their articular cartilage damage.

Objective To investigate the prevalence of ankle sprains and the relationships between recurrence, perceived instability, ATFL pathology, range of motion and the balance test among elite high school basketball players.

Design Cross-sectional study.

Setting High school national championship tournament in 2017.

Patients (or Participants) All players who voluntarily participated in our checkup.

Interventions (or Assessment of Risk Factors) Questionnaire, ultrasonographic examination for anterior talofibular ligament (ATFL) pathology, ankle dorsi-flexion (DF) angle measurement and star excursion balance test (SEBT).

Results We received the responses from 1013 players (330 male and 683 female players). It revealed that $74.3 \%$ of males and $82.7 \%$ of females had injured their ankle at least once and recurrence rate was $59.6 \%$ and $63.0 \%$ respectively. $79.3 \%$ of players were injured before 16 years of age. Fiftytwo male and 77 female players completed all examinations. It was revealed that the players who had recurrent sprains suffered more severe ATFL injuries and felt unstable sensation in their ankle compared with the players without sprain. Players who got ATFL tear had smaller DF angle significantly. Additionally, DF angle significantly correlated with the reach of SBET. Logistic regression analysis indicated that ATFL tear and Unstable sensation were significantly associated with recurrent sprains, and multiple regression analysis revealed that DF angle and gender were only variables to predict SEBT length.

Conclusions Prevention of the CAI should be focused on the following 3 points:1) Education for younger, pre-injury age, 2) Treatment just after their first injury with an emphasis on the ATFL healing and range of motion restoration, 3) Appropriate exercises and/or equipment for prevention from recurrence.

\section{ACUTE EFFECTS OF WARMING UP ON ACHILLES TENDON BLOOD FLOW AND STIFFNESS}

Dries Pieters, Evi Wezenbeek, Roel De Ridder, Erik Witvrouw, Tine Willems. Department of Rehabilitation Sciences and Physiotherapy, Ghent University, Ghent, Belgium., Ghent, Belgium

\subsection{6/bjsports-2021-IOC.105}

Background The influence of warm-up exercises on the muscle properties has already been investigated before. Strikingly, it still remains unclear which warm-up exercises should be performed in order to properly prepare the Achilles tendon in withstanding high-forces during sport participation.

Objective To investigate the acute effect of frequently used warm-up exercises on the Achilles tendon blood flow and stiffness.

Design A within-subject repeated-measure design.

Setting Knowledge about which exercises are suitable to prepare the athlete's Achilles tendon in withstanding high amounts of loading during sport activities could help sport physicians and physiotherapists when recommending warm-up exercises that are able to improve sport performance while reducing the injury susceptibility. This study was conducted at the department of Rehabilitation Sciences at Ghent University. Participants All 40 participants performed at least 1 hour of weekly sports participation and were excluded from this study if they had a history of lower extremity injuries within the previous year.

Assessment Achilles tendon blood flow and stiffness measurements were obtained before and immediately after four different warm-up exercises: running, plyometrics, eccentric heel drops and static stretching. The effect of these warm-up exercises and possible covariates on the Achilles tendon blood flow and stiffness was investigated with linear mixed models.

Main Outcome Measurements Achilles tendon blood flow and stiffness.

Results The results showed a significant increase in Achilles tendon blood flow and stiffness after 10 minutes of running $(\mathrm{p}<0.001$ and $\mathrm{p}<0.001)$ and plyometrics $(\mathrm{p}<0.001$ and $\mathrm{p}=0.039)$. Static stretching and eccentric exercises elicited no significant changes.

Conclusions It could be suggested that warm-up exercises should be intensive enough in order to properly prepare the Achilles tendon for subsequent sport activities. When looking at Achilles tendon blood flow and stiffness, we advise the incorporation of highly intensive exercises such as running and plyometrics within warm-up programs.

\section{THE USE OF INERTIAL MEASUREMENT UNITS FOR ANALYZING CHANGE OF DIRECTION MOVEMENT IN SPORTS: A SCOPING REVIEW}

${ }^{1}$ Aki-Matti Alanen, 'Anu Raisanen, 'Lauren Benson, 1,2,3,4 Kati Pasanen. 'Sport Injury Prevention Research Center, Faculty of Kinesiology, University of Calgary, Calgary, Canada; ${ }^{2}$ Alberta Children's Hospital Research Institute, University of Calgary, Calgary, Canada; ${ }^{3}$ McCaig Institute for Bone and Joint Health, University of Calgary, Calgary, Canada;

${ }^{4}$ Tampere Research Center of Sports Medicine, UKK Institute, Tampere, Finland

\subsection{6/bjsports-2021-IOC.106}

Background Research on change of direction movement (COD) has focused on factors related to performance and injury prevention or rehabilitation. Wearable devices are used 